\title{
LIPSCHITZ HARMONIC CAPACITY AND BILIPSCHITZ IMAGES OF CANTOR SETS
}

\author{
John Garnett, Laura Prat and Xavier Tolsa
}

\begin{abstract}
For bilipschitz images of Cantor sets in $\mathbb{R}^{d}$ we estimate the Lipschitz harmonic capacity and prove that this capacity is invariant under bilipschitz homeomorphisms. A crucial step of the proof is an estimate of the $L^{2}$ norms of the Riesz tranforms on $L^{2}(G, p)$ where $p$ is the natural probability measure on the Cantor set $E$ and $G \subset E$ has $p(G)>0$.
\end{abstract}

\section{Introduction}

Let $L i p_{\text {loc }}^{1}$ be the set of locally Lipschitz real functions on Euclidean space $\mathbb{R}^{d}$, let $E$ be a compact subset of $\mathbb{R}^{d}$, and let

$$
L(E, 1)=\left\{f \in \operatorname{Lip}_{\text {loc }}^{1}: \operatorname{supp}(\Delta f) \subset E,\|\nabla f\|_{\infty} \leq 1 ; \nabla f(\infty)=0\right\}
$$

be the set of locally Lipschitz functions harmonic on $\mathbb{R}^{d} \backslash E$ and normalized by the conditions $\|\nabla f\|_{\infty} \leq 1$ and $\nabla f(\infty)=0$. The Lipschitz harmonic capacity of $E$ is defined by

$$
\kappa(E)=\sup \{|\langle\Delta f, 1\rangle|: f \in L(E, 1)\} .
$$

It was introduced by Paramonov $[\mathrm{P}]$ to study problems of $C^{1}$ approximation by harmonic functions in $\mathbb{R}^{d}$.

If $d=2$ and if the Hausdorff measure $\Lambda_{2}(E)=0$, then $f \in L(E, 1)$ if and only if $F(z)=f_{x}-i f_{y}$ is an analytic function on $\mathbb{C} \backslash E$ such that $\bar{\partial} F$ is real and $|F(z)| \leq 1$. In that case it then follows from Green's theorem that $\kappa(E)=2 \pi \gamma_{\mathbb{R}}(E)$, where

$$
\gamma_{\mathbb{R}}(E)=\sup \left\{\left|\lim _{z \rightarrow \infty} z F(z)\right|: F \text { is analytic on } \mathbb{C} \backslash E,|F| \leq 1, F(\infty)=0, \bar{\partial} F \text { real }\right\}
$$

is the so called real analytic capacity of $E$. (See $[\mathrm{P}]$.) Moreover, by the main result of $[\mathrm{T} 1], \gamma_{\mathbb{R}}(E) \leq \gamma(E) \leq C \gamma_{\mathbb{R}}(E)$ where $\gamma$ is the analytic capacity of $E$ and $C$ is a constant.

Now let $T: \mathbb{R}^{d} \rightarrow \mathbb{R}^{d}$ be a bilipschitz homeomorphism:

$$
A^{-1}|x-y| \leq|T x-T y| \leq A|x-y| .
$$

This paper is concerned with the following conjecture.

Conjecture 1.1. If $T$ is a bilipschitz homeomorphism, then

$$
\kappa(T(E)) \leq C(A) \kappa(E),
$$

where $A$ is the constant in (1).

Received by the editors May 26, 2006. 
When $d=2$ this conjecture was established in [T2] using the connection between analytic capacity and Menger curvature obtained in [T1]. The papers [T1] and [T2] were preceded by two papers $[\mathrm{MTV}]$ and $[\mathrm{GV}]$ that estimated the analytic capacity of planar Cantor sets and of their bilipschitz images. The recent paper [MT] estimated the Lipschitz harmonic capacity of certain Cantor sets in $\mathbb{R}^{d}$, and our purpose here is to establish Conjecture 1.1 for bilipschitz images of these Cantor sets. Thus in the language of fractions, this paper is to $[\mathrm{MT}]$ as paper $[\mathrm{GV}]$ was to $[\mathrm{MTV}]$ or paper [T2] was to [T1].

For fixed ratios $\lambda_{n}$ such that

$$
2^{-\frac{d}{d-1}} \leq \lambda_{n} \leq \lambda_{0}<\frac{1}{2}
$$

we write

$$
\sigma_{n}=\prod_{k=0}^{n} \lambda_{k}
$$

and define the sets

$$
E=\bigcap_{n=0}^{\infty} E_{n}, E_{n}=\bigcup_{|J|=n} Q_{J}^{n},
$$

where $J=\left(j_{1}, j_{2}, \ldots, j_{n}\right)$ is a multi-index of length $n$ with $j_{k} \in\left\{1,2, \ldots 2^{d}\right\}$ and the $Q_{J}^{n}$ are compact sets such that

$$
Q_{\left(J, j_{n+1}\right)}^{n+1} \subset Q_{J}^{n}, \text { for all } n \text { and } J,
$$

and such that for all $n$ and $J$,

$$
c_{1} \sigma_{n} \leq \operatorname{diam}\left(Q_{J}^{n}\right) \leq c_{2} \sigma_{n},
$$

and

$$
\operatorname{dist}\left(Q_{J}^{n}, Q_{K}^{n}\right) \geq c_{3} \sigma_{n}, J \neq K .
$$

for positive constants $c_{1}, c_{2}$, and $c_{3}$.

When $Q_{J}^{n}$ is a cube with sides parallel to the coordinate axes and side-length $\sigma_{n}$ and

$$
\left\{Q_{\left(J, j_{n+1}\right)}^{n+1} \subset Q_{J}^{n}: j_{n+1}=1, \ldots, 2^{d}\right\}
$$

consists of the $2^{d}$ corner subcubes of $Q_{J}^{n}$, the set defined by (3) is the Cantor set studied in [MT], and a set $E$ is the bilipschitz image of such a Cantor set if and only if $E$ satisfies (3), (4), and (5). Write

$$
\theta_{n}=\frac{2^{-n d}}{\sigma_{n}^{d-1}}
$$

and $\theta(Q)=\theta_{n}$ if $Q=Q_{J}^{n}$. Note that by (2),

$$
\theta_{n+1} \leq \theta_{n}
$$

For Cantor sets it was proved in $[\mathrm{MT}]$ that

$$
C^{-1}\left(\sum_{n=0}^{\infty} \theta_{n}^{2}\right)^{-\frac{1}{2}} \leq \kappa(E) \leq C\left(\sum_{n=0}^{\infty} \theta_{n}^{2}\right)^{-\frac{1}{2}}
$$


where $C$ depends only on the constant $\lambda_{0}$ in (2) and we extend their result to bilipschitz images of Cantor sets.

Theorem 1.2. If $E$ is defined by (3), (4), and (5), then there is constant

$$
C=C\left(c_{1}, c_{2}, c_{3}, \lambda_{0}\right)
$$

such that

$$
C^{-1}\left(\sum_{n=1}^{\infty} \theta_{n}^{2}\right)^{-\frac{1}{2}} \leq \kappa(E) \leq C\left(\sum_{n=1}^{\infty} \theta_{n}^{2}\right)^{-\frac{1}{2}}
$$

The proof of Theorem 1.2 follows the reasoning in [MT], but with certain changes. In Section 2 we give some needed geometric properties of the sets $E$. In Section 3 we obtain $L^{2}$ estimates for the (truncated) Riesz transforms with respect to the probability measure $p$ on $E$ defined by $p\left(Q_{J}^{n}\right)=2^{-n d}$ but restricted to a subset $G \subset E$ with $p(G)>0$. In Section 4 we derive Theorem 1.2 from the $L^{2}$-estimates in section 3 by applying the dyadic $T(b)$ Theorem of M. Christ to a measure used in $[\mathrm{MTV}]$ and $[\mathrm{MT}]$.

\section{The geometry of $E$}

Fix $E$ such that (2) - (5) hold.

Lemma 2.1. There is $c_{4}=c_{4}\left(\lambda_{0}, c_{1}, c_{2}, c_{3}\right)$ such that for $j=1,2, \ldots, d$, and all $Q_{J}^{n}$

$$
\sup _{Q_{J}^{n} \cap E} x_{j}-\inf _{Q_{J}^{n} \cap E} x_{j} \geq c_{4} \sigma_{n} .
$$

Proof. Write

$$
w=\sup _{Q_{J}^{n} \cap E} x_{j}-\inf _{Q_{J}^{n} \cap E} x_{j} .
$$

Let $\mathcal{P}$ be the hyperplane

$$
x_{j}=\frac{1}{2}\left(\sup _{Q_{J}^{n} \cap E} x_{j}+\inf _{Q_{J}^{n} \cap E} x_{j}\right),
$$

and let $\tilde{Q}_{K}^{k}$ be the orthogonal projection of $Q_{K}^{k}$ onto $\mathcal{P}$. If

$$
w<\frac{c_{3}}{2} \sigma_{n+p}
$$

then for $k=n+1, \cdots, n+p,(5)$ and the Pythagorean Theorem give

$$
\operatorname{dist}\left(\tilde{Q}_{J^{\prime}}^{k}, \tilde{Q}_{J^{\prime \prime}}^{k}\right) \geq \frac{\sqrt{3}}{2} c_{3} \sigma_{k}
$$

when $\tilde{Q}_{J^{\prime}}^{k} \cup \tilde{Q}_{J^{\prime \prime}}^{k} \subset Q_{J}^{n}$. Consequently there are $(d-1)$-dimensional balls $B_{J^{\prime}}^{k}$ with diameter comparable to the diameter of $\tilde{Q}_{J^{\prime}}^{k}$ such that

$$
\operatorname{dist}\left(\tilde{Q}_{J^{\prime}}^{k}, B_{J^{\prime}}^{k}\right) \leq \frac{\sqrt{3}}{4} c_{s} \sigma_{k}
$$

and

$$
B_{J}^{k} \cap B_{K}^{m}=\varnothing, \text { when } k \geq m .
$$


Hence for constants $c_{5}>c_{6}$ depending only on $d$ and $c_{1}, c_{2}$, and $c_{3}$,

$$
\begin{aligned}
c_{5} \sigma_{n}^{d-1} & \geq \Lambda_{d-1}\left(\bigcup_{k=1}^{p} \bigcup_{|K|=k} B_{(J, K)}^{n+k}\right) \\
& =\sum_{k=1}^{p} \sum_{|K|=k} \Lambda_{d-1}\left(B_{(J, K)}^{n+k}\right) \\
& \geq \sum_{k=1}^{p} c_{6} 2^{k d} \sigma_{n+k}^{d-1}
\end{aligned}
$$

and by (2) this can only happen if $p \leq \frac{c_{5}}{c_{6}}$. Thus (6) holds with $c_{4}=c_{3} 2^{\frac{-d}{d-1} \frac{c_{5}}{c_{6}}-1}$.

Define the probability measure $p$ on $E$ by $p\left(Q_{J}^{n}\right)=2^{-n d}$.

Lemma 2.2. There exist $c_{7}, c_{8}$, and $0<\gamma<1$, depending only on $\lambda_{0}, c_{1}, c_{2}$, and $c_{3}$ such that for $j=1,2, \ldots, d$, there exist at least $c_{7} 2^{n}$ disjoint slabs of the form

$$
S_{k}=\left\{a_{k} \leq x_{j} \leq b_{k}\right\}
$$

such that $b_{k}-a_{k} \leq c_{7} \sigma_{n}, p\left(S_{k}\right)<c_{7} \gamma^{n}$, and $p\left(\bigcup S_{k}\right) \geq c_{8}$.

Proof. Condition (4) implies that there exist disjoint slabs $S_{k}$ satisfying all the conditions of the lemma except possibly $p\left(S_{k}\right) \leq c_{7} \gamma^{n}$. However, by Lemma 2.1 there exists $m_{0}$ such that if $m \leq n-m_{0}$, then for each $Q_{J}^{m}$ at most $2^{d}-1$ cubes $Q_{K}^{m+1} \subset$ $Q_{J}^{m}$ can meet $S_{k}$. Hence the number of $Q_{L}^{n}$ with $Q_{L}^{n} \cap S_{k} \neq \varnothing$ does not exceed $\left(2^{d}-1\right)^{\left(n-m_{0}\right)} 2^{d m_{0}}$ and $p\left(S_{k}\right) \leq\left(1-2^{-d}\right)^{n-m_{0}} \leq c_{7} \gamma^{n}$.

\section{The $L^{2}$ estimate}

Let $E$ satisfy properties (2) - (5). For $x \in E$ we define $Q_{x}^{n}=Q_{J}^{n}$ to be the unique $Q_{J}^{n}$ such that $x \in Q_{J}^{n}$. If $f \in L^{2}(p)$ and $j=1,2, \ldots, d$, we define the truncated Riesz transform as

$$
R_{N}^{j} f(x)=\int_{y \notin Q_{x}^{N}} K_{j}(y-x) f(y) d p(y),
$$

where $K_{j}(y-x)=\frac{(y-x)_{j}}{|y-x|^{d}}$. By (5) it is clear that $\left\|R_{N}^{j}\right\|_{L^{2}(p)}<\infty$.

Theorem 3.1. Let $0<\alpha<1$ and let $G \subset E$ be a closed set such that $p(G)>\alpha$. There are constants $C_{1}(\alpha)$ and $C_{2}$, both depending on $\lambda_{0}, c_{1}, c_{2}$ and $c_{3}$, such that for all $N$ big enough,

$$
C_{1}\left(\sum_{n=0}^{N} \theta_{n}^{2}\right)^{\frac{1}{2}} \leq\left\|R_{N}^{j}\right\|_{L^{2}(G, p)} \leq C_{2}\left(\sum_{n=0}^{N} \theta_{n}^{2}\right)^{\frac{1}{2}} .
$$


To begin we prove the upper bound in (7). Since the norm $\left\|R_{N}^{j}\right\|_{L^{2}(G, p)}$ increases with $G$ we may assume $G=E$, which also means $C_{2}$ does not depend on $\alpha$. The proof of the upper bound in (7) follows the paper [MT], but for convenience we repeat their argument. By the T(1)-Theorem for spaces of homogeneous type from [Ch1] we have

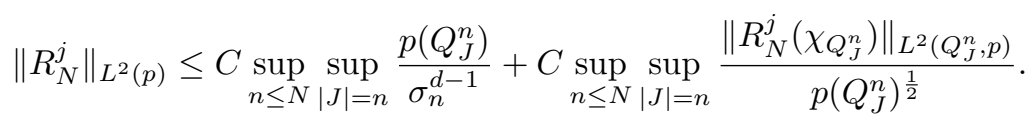

Therefore the upper bound in (7) will be an immediate consequence of the following two lemmas. For convenience we fix $j$, write $K(y-x)=K_{j}(y-x)$, and define

$$
R_{m} f(x)=\int_{Q_{x}^{m} \backslash Q_{x}^{m+1}} K_{j}(y-x) f(y) d p(y) .
$$

Lemma 3.2. If $n \leq m$, there is $c_{9}$ such that

$$
\left\|R_{m} \chi_{Q_{J}^{n}}\right\|_{L^{2}\left(Q_{J}^{n}, p\right)} \leq c_{9} \theta_{m} p\left(Q_{J}^{n}\right)^{\frac{1}{2}}
$$

Proof. For $y \in Q_{x}^{m} \backslash Q_{x}^{m+1}$, (5) gives

$$
|K(y-x)| \leq \frac{1}{c_{3}^{d-1} \sigma_{m+1}^{d-1}}
$$

Hence by (2)

$$
\left|R_{m} \chi_{Q_{J}^{n}}\right| \leq \frac{2^{d}}{c_{3}^{d-1}} \theta_{m}
$$

and

$$
\left\|R_{m} \chi_{Q_{J}^{n}}\right\|_{L^{2}\left(Q_{J}^{n}, p\right)} \leq \frac{2^{d}}{c_{3}{ }^{d-1}} \theta_{m} p\left(Q_{J}^{n}\right)^{\frac{1}{2}}
$$

Lemma 3.3. There is a constant $C$ depending only on $\lambda_{0}, c_{1}, c_{2}$ and $c_{3}$ such that for all $N>n$ and all $J$,

$$
\left\|R_{N}^{j} \chi Q_{J}^{n}\right\|_{L^{2}\left(Q_{J}^{n}, p\right)}^{2} \leq C \sum_{k=n}^{N} \theta_{k}^{2} p\left(Q_{J}^{n}\right) .
$$

Proof. Fix $j=1, \ldots, d$, then for $x \in Q_{J}^{n}$

$$
R_{N}^{j} \chi_{Q_{J}^{n}}(x)=\sum_{m=n}^{N-1} R_{m} \chi_{Q_{J}^{n}}(x) .
$$

We claim that for $m \neq k$,

$$
\left|\int R_{m} \chi_{Q_{J}^{n}} R_{k} \chi_{Q_{J}^{n}} d p\right| \leq C 2^{-|m-k|} \theta_{m} \theta_{k} p\left(Q_{J}^{n}\right) .
$$


Accepting (8) for the moment, we see from Lemma 3.2 that

$$
\begin{aligned}
\left\|R_{N}^{j} \chi_{Q_{J}^{n}}\right\|_{L^{2}\left(Q_{J}^{n}\right)}^{2} & =\left\|\sum_{m=n}^{N-1} R_{m} \chi_{Q_{J}^{n}}\right\|_{L^{2}\left(Q_{J}^{n}\right)}^{2} \\
& =\sum_{m=n}^{N-1}\left\|R_{m} \chi_{Q_{J}^{n}}\right\|_{L^{2}\left(Q_{J}^{n}\right)}^{2}+2 \sum_{n \leq k<m \leq N-1}\left\langle R_{m} \chi_{Q_{J}^{n}}, R_{k} \chi_{Q_{J}^{n}}\right\rangle \\
& \leq C \sum_{m=n}^{N-1} \theta_{m}^{2} p\left(Q_{J}^{n}\right),
\end{aligned}
$$

which gives the right-hand inequality in (7).

To prove (8) assume $n \leq k<m \leq N-1$. Then because the kernel $K$ is odd,

$$
\int_{Q_{K}^{m}} R_{m} \chi_{Q_{J}^{n}}(x) d p(x)=\sum_{r \neq q} \int_{Q_{(K, r)}^{m+1}} \int_{Q_{(K, q)}^{m+1}} K(x-y) d p(y) d p(x)=0,
$$

so that for any $x_{K}^{m} \in Q_{K}^{m}$,

$$
\int_{Q_{K}^{m}} R_{m} \chi_{Q_{J}^{n}}(x) R_{k} \chi_{Q_{J}^{n}}(x) d p(x)=\int_{Q_{K}^{m}} R_{m} \chi_{Q_{J}^{n}}(x)\left(R_{k} \chi_{Q_{J}^{n}}(x)-R_{k} \chi_{Q_{J}^{n}}\left(x_{K}^{m}\right)\right) d p(x) .
$$

But when $x \in Q_{K}^{m},(4),(5)$ and (2) give

$$
\left|R_{k} \chi_{Q_{J}^{n}}(x)-R_{k} \chi_{Q_{J}^{n}}\left(x_{K}^{m}\right)\right| \leq C \frac{\sigma_{m} p\left(Q_{x}^{k}\right)}{\sigma_{k}^{d}} \leq C \theta_{k} \frac{\sigma_{m}}{\sigma_{k}} \leq C 2^{-(m-k)} \theta_{k} .
$$

Hence using Lemma 3.2

$$
\begin{aligned}
\left|\int R_{m} \chi_{Q_{J}^{n}} R_{k} \chi_{Q_{J}^{n}} d p\right| & \leq C 2^{-(m-k)} \theta_{k}\left\|R_{m} \chi_{Q_{J}^{n}}\right\|_{L^{1}\left(Q_{J}^{n}, p\right)} \\
& \leq C 2^{-(m-k)} \theta_{k} p\left(Q_{J}^{n}\right)^{\frac{1}{2}} \theta_{m} p\left(Q_{J}^{n}\right)^{\frac{1}{2}}
\end{aligned}
$$

and (8) holds.

The proof of the lower bound in (7) also follows [MT] but with two alterations needed because $G \neq E$ and because the sets $Q_{J}^{n}$ may be incongruent. When $Q=Q_{J}^{n}$ we also write $n=n(Q), Q \in \mathcal{D}_{n}$, and $\theta(Q)=\theta_{n}$.

Let $0<\delta<1$, fix $G$ and define $\mathcal{B}(\delta)=\left\{Q \in \bigcup_{n} \mathcal{D}_{n}: p(G \cap Q)<\delta p(Q)\right\}$.

Lemma 3.4. Assume $\delta<\alpha$ and $p(G) \geq \alpha$.

(a) Then for all $n$,

$$
p\left(G \backslash \bigcup_{\mathcal{D}_{n} \cap \mathcal{B}(\delta)} Q_{n}^{J}\right) \geq p\left(G \backslash \bigcup_{\mathcal{B}(\delta)} Q\right) \geq \alpha-\delta .
$$

(b) For $N_{0} \in \mathbb{N}$ there exists $M\left(N_{0}\right)$ such that whenever $Q \notin \mathcal{B}(\delta)$, there exist $Q^{\prime} \subset Q$ with $n\left(Q^{\prime}\right) \leq n(Q)+M$ such that for all $Q^{\prime \prime} \subset Q^{\prime}$ with $n\left(Q^{\prime \prime}\right) \leq n\left(Q^{\prime}\right)+N_{0}$

$$
Q^{\prime \prime} \notin \mathcal{B}\left(\frac{\delta}{2}\right) \text {. }
$$


Proof. To prove (a) let $\left\{Q_{j}\right\}$ be a family of maximal cubes in $\mathcal{B}(\delta)$, note that

$$
p\left(G \cap \bigcup_{\mathcal{B}(\delta)} Q\right) \leq \sum p\left(G \cap Q_{j}\right) \leq \delta p(E)=\delta
$$

and subtract this quantity from $p(G)$.

To prove (b) fix $N_{0}$ and suppose (b) is false for $N_{0}, \delta, Q$ and $M=0$. Write $n=n(Q)$. Then there is $Q_{1} \subset Q$ with $n\left(Q_{1}\right) \leq n+N_{0}$ and $Q_{1} \in \mathcal{B}\left(\frac{\delta}{2}\right)$. Set $\mathcal{F}_{1}=\left\{Q_{1}\right\}$. Then $p\left(Q \backslash Q_{1}\right) \leq\left(1-2^{-N_{0} d}\right) p(Q)=\beta p(Q)$. Now assume (b) is also false for $N_{0}, \delta, Q$ and $M=N_{0}$ and write $Q \backslash Q_{1}=\bigcup\left\{Q^{\prime}: n\left(Q^{\prime}\right)=n\left(Q_{1}\right), Q^{\prime} \neq Q_{1}\right\}$. Then for each $Q^{\prime} \neq Q_{1}$ with $n\left(Q^{\prime}\right)=n\left(Q_{1}\right)$ there is $Q_{2} \subset Q^{\prime}$ with $n\left(Q_{2}\right) \leq n+2 N_{0}$ and $Q_{2} \in \mathcal{B}\left(\frac{\delta}{2}\right)$. Set $\mathcal{F}_{2}=\left\{Q_{2}\right\}$. Then $p\left(Q \backslash \bigcup_{\mathcal{F}_{1} \cup \mathcal{F}_{2}} Q_{j}\right) \leq \beta^{2} p(Q)$. Further assume (b) is false for $N_{0}, \delta, Q$ and $M=2 N_{0}$ and repeat the above construction in each $Q^{\prime} \backslash Q_{2}$. After $m$ steps we obtain families $\mathcal{F}_{j}$ of cubes $Q_{j} \in \mathcal{B}\left(\frac{\delta}{2}\right)$ such that $\bigcup \mathcal{F}_{j}$ is disjoint and

$$
p\left(Q \backslash \bigcup_{j=1}^{m} \bigcup_{\mathcal{F}_{j}} Q_{j}\right) \leq \beta^{m} p(Q)
$$

and for $\beta^{m}<\frac{\delta}{2}$ we obtain $p(Q \cap G) \leq \frac{\delta}{2} \sum_{j=1}^{m} \sum_{\mathcal{F}_{j}} p\left(Q_{j}\right)+\beta^{m} p(Q)<\delta p(Q)$, which is a contradiction. We conclude that (b) holds for $M=m N_{0}$.

For any $\delta<\alpha$ we say $Q^{\prime} \in \mathcal{G}^{*}(\delta)$ if $Q^{\prime}$ satisfies conclusion (b) of Lemma 3.4 for $N_{0}$ and $\delta$. Then by parts (b) and (a) of Lemma 3.4 we have:

Lemma 3.5. Let $\delta=\frac{\alpha}{2}$ and assume $p(G) \geq \alpha$. Then

$$
\sum_{\mathcal{G}^{*}\left(\frac{\delta}{2}\right)} \theta\left(Q^{\prime}\right)^{2} p\left(Q^{\prime} \cap G\right) \geq C(M) \sum_{Q \notin \mathcal{B}(\delta)} \theta(Q)^{2} p(Q \cap G) \geq C(M, \alpha) \sum \theta_{n}^{2} .
$$

Now let $A$ be a large constant. As in [MT], for $R \in \mathcal{D}$ we will define a family $\operatorname{Stop}(R)$ of "stopping cubes" $Q \subset R$. We say $Q \in \operatorname{Stop}_{0}(R)$ if $Q \subset R$ and $Q \notin \mathcal{B}\left(\frac{\delta}{2}\right)$ and if

$$
\inf _{Q}\left|\int_{G \cap(R \backslash Q)} K(y-x) d p(y)\right| \geq A \theta(R) .
$$

We also say $Q \in \operatorname{Stop}_{1}(R)$ if $Q \subset R$ and $Q \notin \mathcal{B}\left(\frac{\delta}{2}\right)$, if $\theta(Q) \leq \eta \theta(R)$ for constant $\eta$ to be chosen below, if $n(Q) \geq n(R)+N_{1}$ for constant $N_{1}$ to be chosen below, and if

$$
P \in \operatorname{Stop}_{0}(R) \Rightarrow n(P) \geq n(Q) .
$$

Then define

$$
\operatorname{Stop}(R)=\left\{Q \in \operatorname{Stop}_{0}(R) \cup \operatorname{Stop}_{1}(R): Q \text { is maximal }\right\} .
$$

It follows from the last three conditions in the definition of $\operatorname{Stop}_{1}(R)$ that either $\operatorname{Stop}(R) \subset \operatorname{Stop}_{0}(R)$ or $\operatorname{Stop}(R) \subset \operatorname{Stop}_{1}(R)$. Inductively we define $\operatorname{Stop}^{1}(P)=$ $\operatorname{Stop}(P)$ and

$$
\begin{gathered}
\operatorname{Stop}^{k}(P)=\bigcup\left\{\operatorname{Stop}(Q): Q \in \operatorname{Stop}^{k-1}(P)\right\}, \\
\operatorname{Top}=\left\{P_{0}\right\} \cup \bigcup_{k \geq 1} \operatorname{Stop}^{k}\left(P_{0}\right),
\end{gathered}
$$


where $P_{0}$ is the unique cube in $\mathcal{D}_{0}$, and

$$
P^{s t p}=\bigcup_{\operatorname{Stop}(P)} Q
$$

Remark. The constants $N_{0}, N_{1}, A, \eta$ are chosen as follows. First we take $\delta=\alpha / 2$. Then $N_{1}$ will be determined by Lemma 3.7, $\eta$ and $A$ will be determined by the proof of Lemma 3.8, and $N_{0}$, which depends on $A, \eta$, and $\delta$, will be determined by the proof of Lemma 3.6.

Lemma 3.6. Let $\delta=\frac{\alpha}{2}$ and assume $p(G) \geq \alpha$. If $N_{0}=N_{0}(A, \eta, \delta)$ is sufficiently large, then for all $Q \in \mathcal{G}^{*}\left(\frac{\delta}{2}\right)$ there exists a cube $P \subset Q$ such that $P \in$ Top and $n(P) \leq n(Q)+N_{0}$.

Proof. Let $Q \in \mathcal{G}^{*}\left(\frac{\delta}{2}\right)$ and let $R$ be the smallest cube $R \in$ Top such that $Q \subset R$. We assume the conclusion of the lemma is false for $Q$. Thus $Q \notin \operatorname{Top}$, and $Q \notin \operatorname{Stop}(R)$. Hence by definition there is $x_{0} \in Q$ such that

$$
\left|\int_{G \cap R \backslash Q} K\left(y-x_{0}\right) d p(y)\right| \leq A \theta(R) .
$$

Then for $x \in Q(5)$ gives

$$
\left|\int_{G \cap R \backslash Q}\left(K(y-x)-K\left(y-x_{0}\right)\right) d p\right| \leq C \sigma_{n(Q)} \sum_{k=n(R)}^{n(Q)-1} \frac{\theta_{k}}{\sigma_{k}} \leq C_{1} \theta(R)
$$

so that

$$
\sup _{Q}\left|\int_{G \cap R \backslash Q} K(y-x) d p(y)\right| \leq\left(A+C_{1}\right) \theta(R) .
$$

Take $x^{*} \in Q \cap E$ with $x_{j}^{*}=\inf _{Q} x_{j}$ and let $Q^{*}$ be that $Q^{*} \subset Q$ such that $x^{*} \in Q^{*}$ and $n\left(Q^{*}\right)=n(Q)+N_{0}$. Then

$$
K\left(y-x^{*}\right) \geq 0
$$

for all $y \in Q$ and by Lemma 2.1 there is a constant $n_{0}$ such that if $n \leq n\left(Q^{*}\right)-n_{0}$, there exists $Q_{J}^{n} \subset\left(Q \backslash Q^{*}\right)$ such that

$$
\inf _{y \in Q_{J}^{n}} K\left(y-x^{*}\right) \geq \frac{c}{\sigma_{n}^{d-1}} .
$$

Because $\theta_{n+1} \leq \theta_{n}$ and because we assume the lemma is false for $Q$, we also have $\theta\left(Q_{J}^{n}\right) \geq \eta \theta(R)$ for every such $Q_{J}^{n}$. Hence by (5)

$$
\int_{G \cap Q \backslash Q^{*}} K\left(y-x^{*}\right) d p(y) \geq\left(N_{0}-n_{0}\right) \eta \frac{\delta}{2} \theta(R)
$$

and by the proof of (9),

$$
\inf _{Q^{*}} \int_{G \cap Q \backslash Q^{*}} K(y-x) d p(y) \geq\left(\left(N_{0}-n_{0}\right) \eta \frac{\delta}{2}-C\right) \theta(R) .
$$

Taking $N_{0}=N_{0}(A)$ sufficiently large and comparing (10) with (9) we conclude that $Q^{*} \in \operatorname{Stop}_{0}(R)$, which is a contradiction. 
Note that by Lemma 3.5 and Lemma 3.6 we have for all $P$,

$$
\sum_{n=0}^{N} \theta_{n}^{2} \leq C(\alpha) \sum_{n=0}^{N} \sum_{\mathcal{D}_{n} \backslash \mathcal{B}(\delta)} \theta(Q)^{2} p(Q) \leq C^{\prime}(\alpha) \sum_{\text {Top }} \theta(P)^{2} p(G \cap P) .
$$

We define

$$
\begin{aligned}
K_{P} 1(x) & =\sum_{Q \in \operatorname{Stop}(P)} \chi_{G \cap Q}(x) \int_{G \cap P \backslash Q} K(y-x) d p(y) \\
& +\chi_{G \cap P \backslash P^{s t p}}(x) \int_{G \cap P \backslash Q^{N}(x)} K(y-x) d p(y) .
\end{aligned}
$$

By construction

$$
\chi_{G} R_{N} 1=\sum_{\text {Top }} K_{P} 1
$$

and

$$
\left\|R_{N} 1\right\|_{L^{2}(G)}^{2}=\sum_{\text {Top }}\left\|K_{P} 1\right\|_{L^{2}(G)}^{2}+\sum_{P, Q \in \operatorname{Top}, P \neq Q}\left\langle K_{P} 1, K_{Q} 1\right\rangle_{L^{2}(G)} .
$$

Lemma 3.7. If $N_{1}$ is chosen big enough, then for all $P \in$ Top,

$$
\left\|K_{P} 1\right\|_{L^{2}(G)}^{2} \geq C^{-1} \theta(P)^{2} p(G \cap P),
$$

where $C=C(\alpha)$, and

$$
\left\|K_{P} 1\right\|_{L^{2}(G)}^{2} \geq A^{2} \theta(P)^{2} p\left(G \cap P^{s t p_{0}}\right),
$$

where

$$
P^{s t p_{0}}=\bigcup\left\{Q: Q \in \operatorname{Stop}(P) \cap \operatorname{Stop}_{0}(P)\right\} .
$$

\section{Lemma 3.8.}

$$
\sum_{P, Q \in \operatorname{Top}, P \neq Q}\left|\left\langle K_{P} 1, K_{Q} 1\right\rangle_{L^{2}(G)}\right| \leq C\left(A^{-1}+c(\eta)\right) \sum_{\text {Top }}\left\|K_{P} 1\right\|_{L^{2}(G)}^{2},
$$

with $c(\eta) \rightarrow 0$ as $\eta \rightarrow 0$.

Assuming Lemma 3.7 and Lemma 3.8 for the moment, we see that if $A$ is large and $\eta$ is small, then

$$
\left\|R_{N} 1\right\|_{L^{2}(G)}^{2} \geq C^{-1} \sum_{\text {Top }} \theta(P)^{2} p(G \cap P)
$$

and then the lower bound in (7) follows from inequality (11).

To prove Lemma 3.7, first note that (13) follows from the definitions of $\operatorname{Stop}_{0}(P)$ and $\operatorname{Stop}(P)$. To prove (12), recall that $K=K_{j}$ for some $1 \leq j \leq d$. We apply Lemma 2.2 to $P$ with $\gamma^{n} \sim \alpha$ to obtain sets $S_{1} \subset P$ and $S_{2} \subset P$ such that

$$
\sup _{S_{1}} x_{j}=a<\inf _{S_{2}} x_{j}
$$

and

$$
\operatorname{Min}\left(p\left(G \cap S_{1}\right), p\left(G \cap S_{2}\right)\right) \geq c(\alpha) p(P) .
$$


We may assume that $S_{1}, S_{2}$ are much bigger that any stopping cube of $P$, because if there exists some $Q \in \operatorname{Stop}_{0}(P)$ with size similar to $S_{1}$ or $S_{2}$, then (12) follows from (13); and if we choose $N_{1}$ big enough, any cube $Q \in \operatorname{Stop}_{1}(P)$ will be much smaller that $S_{1}, S_{2}$. Then we get

$$
\left|\int_{S_{2} \cap G} K_{P} \chi_{S_{1}}(x) d p(x)\right| \geq C^{-1} p\left(S_{2} \cap G\right) \frac{p\left(S_{1} \cap G\right)}{\operatorname{diam}(P)^{d-1}} .
$$

Set

$$
E_{1}=P \cap\left\{x_{j} \leq a\right\} \text { and } E_{2}=P \cap\left\{x_{j}>a\right\} .
$$

By its definition,

$$
K_{P} 1=\chi_{G}(x) \sum_{k} \chi_{Q_{k}}(x) \int_{G \cap P \backslash Q_{k}} K(y-x) d p(y)
$$

where $\left\{Q_{k}\right\}$ is a cover of $P$ by disjoint cubes from $\mathcal{D}$. We also have

$$
\begin{aligned}
K_{P} 1(x) & =\chi_{G}(x) \sum_{i=1,2} \sum_{k} \chi_{Q_{k}}(x) \int_{G \cap E_{i} \backslash Q_{k}} K(y-x) d p(y) \\
& \equiv K_{P} \chi_{E_{1}}(x)+K_{P} \chi_{E_{2}}(x) .
\end{aligned}
$$

Write $Q_{k}=Q(x)$ when $x \in Q_{k}$ and note that

$$
y \notin Q(x) \Longleftrightarrow x \notin Q(y) .
$$

Hence by the antisymmetry $K(y-x)=-K(x-y)$ we have

$$
\int_{G \cap E_{2}} K_{P} \chi_{E_{2}}(x) d p(x)=0 .
$$

Therefore by the choices of $S_{1}, S_{2}, E_{1}$ and $E_{2}$,

$$
\begin{aligned}
\left(p\left(G \cap E_{2}\right)\right)^{1 / 2}\left\|K_{P} 1\right\|_{L^{2}(G)} & \geq\left|\int_{G \cap E_{2}} K_{P} 1(x) d p(x)\right| \\
& =\left|\int_{G \cap E_{2}} K_{P} \chi_{E_{1}}(x) d p(x)\right| \\
& \geq\left|\int_{S_{2} \cap G} K_{P} \chi_{S_{1}}(x) d p(x)\right| \\
& \geq p\left(G \cap E_{2}\right) \frac{c(\alpha) p(G \cap P)}{\operatorname{diam}(P)^{d-1}},
\end{aligned}
$$

which is (12).

To prove Lemma 3.8 we again follow [MT]. Suppose $P \neq Q \in$ Top and $Q \subset P$. Let $P_{Q} \in \operatorname{Stop}(P)$ be such that $Q \subset P_{Q} \subset P$. By the antisymmetry of $K$ we have $\int_{Q \cap G} K_{Q} 1 d p=0$ so that

$$
\begin{aligned}
\left|\int_{Q \cap G} K_{Q} 1(x) K_{P} 1(x) d p\right| & =\left|\int_{Q \cap G} K_{Q} 1(x)\left(K_{P} 1(x)-K_{P} 1\left(x_{Q}\right)\right) d p(x)\right| \\
& \leq\left\|K_{Q} 1\right\|_{L^{1}(Q)} \sup _{Q}\left|K_{P} 1(x)-K_{P} 1\left(x_{Q}\right)\right|
\end{aligned}
$$


where $x_{Q}$ is a fixed point from $Q$. But for any $x \in Q$, standard estimates yield

$$
\begin{aligned}
\left|K_{P} 1(x)-K_{P} 1\left(x_{Q}\right)\right| & \leq \int_{G \cap P \backslash P_{Q}}\left|K(y-x)-K\left(y-x_{Q}\right)\right| d p(y) \\
& \leq C \operatorname{diam}(Q) \int_{G \cap P \backslash P_{Q}} \frac{d p(y)}{|x-y|^{d}} \\
& \leq C \operatorname{diam}(Q) \sum_{P_{Q} \subset R \subset P} \frac{\theta(R)}{\operatorname{diam}(R)} .
\end{aligned}
$$

Assume first that $P_{Q} \in \operatorname{Stop}_{0}(P)$. Since $\theta(R) \leq \theta(P)$ in the last sum, we get

$$
\left|K_{P} 1(x)-K_{P} 1\left(x_{Q}\right)\right| \leq C \frac{\operatorname{diam}(Q)}{\operatorname{diam}\left(P_{Q}\right)} \theta(P) .
$$

Hence by (13),

$$
\left|\left\langle K_{P} 1, K_{Q} 1\right\rangle_{L^{2}(G, p)}\right| \leq \frac{C}{A} \frac{\operatorname{diam}(Q)}{\operatorname{diam}\left(P_{Q}\right)}\left(\frac{p(G \cap Q)}{p\left(G \cap P^{s t p_{0}}\right)}\right)^{1 / 2}\left\|K_{Q} 1\right\|_{L^{2}(G)}\left\|K_{P} 1\right\|_{L^{2}(G)},
$$

when $P_{Q} \in \operatorname{Stop}_{0}(P)$.

Consider now the case $P_{Q} \in \operatorname{Stop}_{1}(P)$. This means that $\theta\left(P_{Q}\right) \leq \eta \theta(P)$. Then it follows from (2) that

$$
\sum_{P_{Q} \subset R \subset P}\left\{\frac{\theta(R)}{\theta(P)}: \frac{\operatorname{diam}\left(P_{Q}\right)}{\operatorname{diam}(R)} \geq c_{1}(\eta)\right\} \leq c_{2}(\eta) \sum_{P_{Q} \subset R \subset P} \frac{\theta(R)}{\theta(P)}
$$

so that

$$
\operatorname{diam}(Q) \sum_{P_{Q} \subset R \subset P} \frac{\theta(R)}{\operatorname{diam}(R)} \leq c(\eta) \frac{\operatorname{diam}(Q)}{\operatorname{diam}\left(P_{Q}\right)} \theta(P) \quad \text { with } c(\eta) \rightarrow 0 \text { as } \eta \rightarrow 0 .
$$

Hence by (12),

$$
\left|\left\langle K_{P} 1, K_{Q} 1\right\rangle_{L^{2}(G, p)}\right| \leq c(\eta) \frac{\operatorname{diam}(Q)}{\operatorname{diam}\left(P_{Q}\right)}\left\|K_{Q} 1\right\|_{L^{2}(G)}\left\|K_{P} 1\right\|_{L^{2}(G)},
$$

when $P_{Q} \in \operatorname{Stop}_{1}(P)$. Thus (14) follows from Schur's lemma.

\section{Lipschitz harmonic capacity}

In this section we will prove Theorem 1.2. We will assume that each cube $Q_{J}^{n}$ in the definition of the Cantor set $E$ (see (3)) contains a closed ball $B_{J}^{n}$ such that

$$
c_{1}^{\prime} \sigma_{n} \leq \operatorname{diam}\left(B_{J}^{n}\right) .
$$

This assumption comes for free from the definition of $E$ in Section 1. Indeed, one easily deduces that there exists a family of balls $B_{J}^{n}$ centered at $Q_{J}^{n}$ such that

$$
c_{1}^{\prime} \sigma_{n} \leq \operatorname{diam}\left(B_{J}^{n}\right) \leq c_{2}^{\prime} \sigma_{n},
$$

and

$$
\operatorname{dist}\left(B_{J}^{n}, B_{K}^{n}\right) \geq c_{3}^{\prime} \sigma_{n}, J \neq K
$$


Then if one replaces the cubes $Q_{J}^{n}$ in the definition of $E$ by the sets

$$
\tilde{Q}_{J}^{n}=\bigcup_{Q_{K}^{m} \subset Q_{J}^{n}}\left(Q_{K}^{m} \cup B_{K}^{m}\right)
$$

$E$ does not change.

Given a real Radon measure $\mu$ and $f \in L^{1}(\mu)$, let

$$
R_{\mu, \epsilon}(f d \mu)(x)=\int_{|y-x|>\epsilon} \frac{y-x}{|y-x|^{d}} f(y) d \mu(y)
$$

be the (truncated) $(d-1)$-Riesz transform of $f \in L^{1}(\mu)$ with respect to the measure $\mu$ and set $\left\|R_{\mu}\right\|_{L^{2}(\mu)}=\sup _{\epsilon>0}\left\|R_{\mu, \epsilon}\right\|_{L^{2}(\mu)}$.

As in [MT], we need to introduce the following capacity of the sets $E_{N}$ :

$$
\kappa_{p}\left(E_{N}\right)=\sup \left\{\alpha: 0 \leq \alpha \leq 1,\left\|R_{\alpha \mu_{N}}\right\|_{L^{2}\left(\alpha \mu_{N}\right)} \leq 1\right\}
$$

where $\mu_{N}$ is a probability measure on $E_{N}$ such that $\mu_{N}\left(Q_{J}^{N}\right)=2^{-N d}$.

The $L^{2}$ estimates from the previous section yield the following lemma.

\section{Lemma 4.1.}

$$
\kappa_{p}\left(E_{N}\right) \approx\left(\sum_{n=1}^{N} \theta_{n}^{2}\right)^{-1 / 2}
$$

Proof. By Theorem 3.1 we have

$$
\left\|R_{\alpha \mu_{N}}\right\|_{L^{2}\left(\alpha \mu_{N}\right)}=\alpha\left\|R_{\mu_{N}}\right\|_{L^{2}\left(\mu_{N}\right)} \approx \alpha\left(\sum_{n=1}^{N} \theta_{n}^{2}\right)^{1 / 2} .
$$

The lemma follows because the sum above is $\geq 2^{-d}$.

We will prove the following:

Lemma 4.2. There exists an absolute constant $C_{0}$ such that for all $N \in \mathbb{N}$ we have

$$
\kappa\left(E_{N}\right) \leq C_{0} \kappa_{p}\left(E_{N}\right)
$$

Notice that Theorem 1.2 follows from Lemma 4.2 and

$$
\kappa\left(E_{N}\right) \geq \kappa_{+}\left(E_{N}\right) \geq C^{-1} \kappa_{p}\left(E_{N}\right),
$$

where

$$
\kappa_{+}(E)=\sup \left\{|\langle\Delta f, 1\rangle|: f \in L(E, 1), \Delta f=\mu \in M_{+}(E)\right\}
$$

and $M_{+}(E)$ is the set of positive Borel measures supported on $E$. The first inequality in (16) is just a consequence of the definitions of $\kappa$ and $\kappa_{+}$and the second inequality follows from a well known method that dualizes a weak $(1,1)$ inequality (see Theorem 23 in [Ch2] and Theorem 2.2 in [MTV]. The original proof is from [D $\varnothing]$ ).

In $[\mathrm{Vo}]$ it is shown that the capacities $\kappa$ and $\kappa_{+}$are comparable for all subsets of $\mathbb{R}^{d}$, but we do not use that deep result.

For any $s>0$, we write $\Lambda_{s}$ and $\Lambda_{s}^{\infty}$ for the $s$-dimensional Hausdorff measure and the $s$-dimensional Hausdorff content, respectively. 
Proof. The arguments are similar to those in $[\mathrm{MTV}]$ and $[\mathrm{MT}]$, but a little more involved because our Cantor sets are not homogeneous. Also, instead of using the local $T(b)$-Theorem of M. Christ, we will run a stopping time argument in the spirit of [Ch1] and then use a dyadic $T(b)$-Theorem (see Theorem 20 in [Ch1]).

We set

$$
S_{n}=\theta_{1}^{2}+\theta_{2}^{2}+\cdots+\theta_{n}^{2}
$$

Without loss of generality we can assume that for each $N>1$ there exists $1 \leq M<N$ such that

$$
S_{M} \leq \frac{S_{N}}{2}<S_{M+1}
$$

Otherwise $\frac{S_{N}}{2}<S_{1}$ and by Lemma 4.1 it follows that $\kappa_{p}\left(E_{N}\right) \geq C^{-1} \lambda_{1}^{d-1}$. By $[\mathrm{P}]$ we have

$$
\kappa\left(E_{N}\right) \leq \kappa\left(E_{1}\right) \leq C \Lambda_{d-1}^{\infty}\left(E_{1}\right) \leq C \lambda_{1}^{d-1},
$$

and if $C_{0}$ is chosen big enough the conclusion of the lemma will follow in this case.

Assuming (17), we will now prove (15) by induction on $N$. For $N=1$ (15) holds clearly. The induction hypothesis is

$$
\kappa\left(E_{n}\right) \leq C_{0} \kappa_{p}\left(E_{n}\right), \text { for } 0<n<N,
$$

where the precise value of $C_{0}$ is to be determined later.

Notice that for $n \geq 0,\left(Q_{K}^{N} \cap E\right)_{n}$ is the $n$-th generation of the Cantor set $Q_{K}^{N} \cap E$, i.e. the union of $2^{n d}$ sets $Q_{J}^{n+N}$ satisfying properties (4) and (5) with $n$ replaced by $n+N$. Let $J^{*}$ be the multi-index of length $M$ such that

$$
\kappa\left(\left(Q_{J^{*}}^{M} \cap E\right)_{N-M}\right)=\max _{|J|=M} \kappa\left(\left(Q_{J}^{M} \cap E\right)_{N-M}\right) .
$$

We distinguish two cases.

Case 1: For some absolute constant $A_{0}$ to be determined below,

$$
\kappa\left(\left(Q_{J^{*}}^{M} \cap E\right)_{N-M}\right) \geq A_{0} 2^{-M d} \kappa\left(E_{N}\right),
$$

By the induction hypothesis (applied to $\left.\left(Q_{J^{*}}^{M} \cap E\right)_{N-M}\right)$ and by Lemma 4.1 we have that

$$
\begin{gathered}
\kappa\left(E_{N}\right) \leq A_{0}^{-1} 2^{M d} \kappa\left(\left(Q_{J^{*}}^{M} \cap E\right)_{N-M}\right) \leq A_{0}^{-1} 2^{M d} C_{0} \kappa_{p}\left(\left(Q_{J^{*}}^{M} \cap E\right)_{N-M}\right) \\
\leq A_{0}^{-1} C_{0} C 2^{M d}\left(\sum_{n=1}^{N-M}\left(\frac{2^{-d n}}{\sigma_{M+n}^{d-1}}\right)^{2}\right)^{-1 / 2}=A_{0}^{-1} C_{0} C\left(\sum_{n=M+1}^{N} \theta_{n}^{2}\right)^{-1 / 2} .
\end{gathered}
$$

Now by using that $S_{M} \leq S_{N} / 2$ is equivalent to $\sum_{n=1}^{N} \theta_{n}^{2} \leq 2 \sum_{n=M+1}^{N} \theta_{n}^{2}$ and Lemma 4.1 again, we obtain that

$$
\kappa\left(E_{N}\right) \leq 2^{1 / 2} A_{0}^{-1} C_{0} C\left(\sum_{n=1}^{N} \theta_{n}^{2}\right)^{-1 / 2} \leq C A_{0}^{-1} C_{0} \kappa_{p}\left(E_{N}\right) .
$$

Hence if $A_{0}=C$, we obtain (15).

Case 2: For the same constant $A_{0}$,

$$
\kappa\left(\left(Q_{J^{*}}^{M} \cap E\right)_{N-M}\right) \leq A_{0} 2^{-M d} \kappa\left(E_{N}\right) .
$$


Then if $\theta_{M+1}^{2}>S_{M}, S_{M+1}=S_{M}+\theta_{M+1}^{2} \approx \theta_{M+1}^{2}$. Therefore

$$
\kappa_{p}\left(E_{M+1}\right) \approx S_{M+1}^{-1 / 2} \approx \theta_{M+1}^{-1} \geq C \Lambda_{d-1}^{\infty}\left(E_{M+1}\right) .
$$

Hence by (17),

$$
\kappa\left(E_{N}\right) \leq \kappa\left(E_{M+1}\right) \leq C \Lambda_{d-1}^{\infty}\left(E_{M+1}\right) \leq C \kappa_{p}\left(E_{M+1}\right) \approx \kappa_{p}\left(E_{N}\right),
$$

which is (15) if $C_{0}$ is chosen big enough.

On the other hand, if $\theta_{M+1}^{2} \leq S_{M}$, then $S_{M+1} \approx S_{M} \approx S_{N}$. Recall that we are assuming that each cube $Q_{J}^{M}$ contains some ball $B_{J}^{M}$ with comparable diameter. Moreover, we may suppose that all the balls $B_{J}^{M}, J=1, \ldots, 2^{M d}$, have the same diameter $d_{M}$. We set

$$
\tilde{E}_{M}=\bigcup_{|J|=M} B_{J}^{M}
$$

We consider now the measure

$$
\sigma=\kappa\left(E_{N}\right) \mu_{M}^{\prime}
$$

where $\mu_{M}^{\prime}$ is defined by

$$
\mu_{M}^{\prime}(K)=\sum_{J} \frac{\Lambda_{d-1}\left(K \cap \partial B_{J}^{M}\right)}{\Lambda_{d-1}\left(\partial \tilde{E}_{M}\right)},
$$

for compact sets $K$. Clearly $\sigma\left(\tilde{E}_{M}\right)=\kappa\left(E_{N}\right)$.

Note that the measure $\sigma$ is doubling and has $(d-1)$-growth. To verify this, one uses that

$$
\kappa\left(E_{N}\right) \leq \kappa\left(E_{M}\right) \leq C \Lambda_{d-1}^{\infty}\left(E_{M}\right) \leq C \Lambda_{d-1}\left(\partial \tilde{E}_{M}\right)
$$

and $\mu_{M}^{\prime}\left(Q_{K}^{n}\right)=2^{-n d}$ for all $0 \leq n \leq M$ (see (4.8) and (4.9) of [MT]).

We will show that there exists a good set $G \subset \tilde{E}_{M}$ with $\sigma(G) \approx \sigma\left(\tilde{E}_{M}\right)$ such that $R_{\sigma_{\mid G}}$ is bounded on $L^{2}\left(\sigma_{\mid G}\right)$ with absolute constants. From this fact, by Theorem 3.1 we have

$$
\left\|R_{\sigma_{\mid G}}\right\|_{L^{2}\left(\sigma_{\mid G}\right)} \approx \kappa\left(E_{N}\right) S_{M}^{1 / 2} \leq C .
$$

So by Lemma 4.1 we infer

$$
\kappa\left(E_{N}\right) \leq C S_{M}^{-1 / 2} \leq C S_{N}^{-1 / 2} \approx C \kappa_{p}\left(E_{N}\right),
$$

which proves the lemma.

To establish the existence of the set $G$, we run a stopping time argument. First we construct a set $E^{\prime}$ and a doubling measure $\sigma^{\prime}$ on $E^{\prime}$. The pair $\left(E^{\prime}, \sigma^{\prime}\right)$ is endowed with a system of dyadic cubes $\mathcal{Q}\left(E^{\prime}\right)$, where

$$
\mathcal{Q}\left(E^{\prime}\right)=\left\{Q_{\beta}^{k} \subset E^{\prime}: \beta \in \mathbb{N}, k \in \mathbb{N}\right\}
$$

(see Theorem 11 in [Ch1]). We also define a function $b^{\prime}$ on $E^{\prime}$, dyadic para-accretive with respect to this system of dyadic cubes, i.e. for every $Q_{\beta}^{k} \in \mathcal{Q}\left(E^{\prime}\right)$, there exists $Q_{\gamma}^{l} \in \mathcal{Q}\left(E^{\prime}\right), Q_{\gamma}^{l} \subset Q_{\beta}^{k}$, with $l \leq k+N$ and

$$
\left|\int_{Q_{\gamma}^{l}} b^{\prime} d \sigma^{\prime}\right| \geq c \sigma^{\prime}\left(Q_{\gamma}^{l}\right)
$$

for some fixed constants $c>0$ and $N \in \mathbb{N}$, and such that the function $R\left(b^{\prime} d \sigma^{\prime}\right)$ belongs to dyadic $\mathrm{BMO}\left(\sigma^{\prime}\right)$. Therefore, the $(d-1)$-Riesz transform $R$ associated to 
$\sigma^{\prime}$ will be bounded on $L^{2}\left(E^{\prime}, \sigma^{\prime}\right)$ by the $T(b)$-theorem on a space of homogeneous type (see Theorem 20 in [Ch1]). Our set $G$ will be contained in $E^{\prime} \cap \tilde{E}_{M}$.

Now we turn to the construction of the set $E^{\prime}$ and the measure $\sigma^{\prime}$. By definition there exists a distribution $T$ supported on $E_{N}$ such that

$$
\kappa\left(E_{N}\right) \leq C|\langle T, 1\rangle|
$$

and

$$
\|R T\|_{L^{\infty}\left(\mathbb{R}^{d}\right)} \leq 1
$$

We replace the distribution $T$ with a real measure $\nu$ supported on $E_{N}$ such that $\kappa\left(E_{N}\right) \leq C\left|\nu\left(E_{N}\right)\right|$ and $\|R \nu\|_{L^{\infty}}\left(\mathbb{R}^{d}\right) \leq 1$. (The measure $\nu$ exists because of Volberg's theorem ([Vo]), but in the special case of $E_{N}$ considered here $\nu$ can be constructed directly by setting $\nu=\sum_{|J|=N} \nu_{J}$ with $\nu_{J}=h_{J} \chi_{\partial B_{J}^{N}} \Lambda_{d-1}$ and $h_{J}$ smooth on $\partial B_{J}^{N}$ such that for all polynomials $P$ of degree at most $d, \int P(x) d \nu_{J}=T\left(P(x) \varphi_{J}(x)\right)$, where $\varphi_{J}$ is smooth and $\varphi_{J}=\chi_{Q_{J}^{N}}$ on $E_{N}$. See $[\mathrm{P}]$.)

The definition of $\sigma$ implies that

$$
\left|\nu\left(E_{N}\right)\right| \geq C^{-1} \sigma\left(\tilde{E}_{M}\right)>\epsilon_{0} \sigma\left(\tilde{E}_{M}\right),
$$

where $\epsilon_{0}$ is a sufficiently small constant to be fixed later. Notice that for a fixed generation $n, 0 \leq n \leq M$, there exists at least one cube $Q_{K}^{n}$, such that $\left|\nu\left(Q_{K}^{n}\right)\right|>$ $\epsilon_{0} \sigma\left(Q_{K}^{n}\right)$, since otherwise for $0 \leq n \leq M$

$$
\left|\nu\left(E_{N}\right)\right| \leq \sum_{|K|=n} \epsilon_{0} \sigma\left(Q_{K}^{n}\right)=\epsilon_{0} \sum_{|J|=M} \sigma\left(B_{J}^{M}\right)=\epsilon_{0} \sigma\left(\tilde{E}_{M}\right),
$$

which contradicts (19).

We now run a stopping-time procedure. Let $\epsilon>0$ be another constant to be chosen later, much smaller than $\epsilon_{0}$. We check whether or not the condition

$$
\left|\nu\left(Q_{J}^{1}\right)\right| \leq \epsilon \sigma\left(Q_{J}^{1}\right)
$$

holds for the cubes $Q_{J}^{1}$. If (20) holds for the cube $Q_{J}^{1}$, we call it stopping-time cube. If (20) does not hold for $Q_{J}^{1}$, we consider the children $Q_{K}^{2}$ of $Q_{J}^{1}$ and call each such $Q_{K}^{2}$ with (20) a stopping-time cube. We continue this procedure through generation $M$, but we do not consider the cubes of later generations. We obtain in this way a collection of pairwise disjoint stopping-time cubes $\left\{P_{\gamma}\right\}_{\gamma}$, where $P_{\gamma}=Q_{J}^{n}$, for some $0 \leq n \leq M$ and by definition each $P_{\gamma}$ satisfies condition (20) with $Q_{J}^{1}$ replaced by $P_{\gamma}$.

Consider now the function

$$
b=\sum_{|J|=M} \frac{\nu\left(Q_{J}^{M}\right)}{\sigma\left(B_{J}^{M}\right)} \chi_{B_{J}^{M}} .
$$

The function $b$ has the following three important properties:

(1) for $0 \leq n \leq M, \int_{Q_{K}^{n}} b d \sigma=\nu\left(Q_{K}^{n}\right)$.

(2) $\|b\|_{\infty} \leq C$.

(3) For any $0 \leq n \leq M$,

$$
\left\|R\left(b \chi_{Q_{K}^{n}} d \sigma\right)\right\|_{L^{\infty}\left(\mathbb{R}^{d}\right)} \leq C .
$$


To show that $b$ is bounded it is enough to verify that

$$
\left|\nu\left(Q_{J}^{M}\right)\right| \leq C \sigma\left(B_{J}^{M}\right) \text {, for }|J|=M
$$

Inequality $(22)$ can be shown by localizing the potential $\nu * x /|x|^{d}$ (see $[\mathrm{P}]$ and $[\mathrm{MPrV}])$ and using (18), namely

$$
\left|\nu\left(Q_{J}^{M}\right)\right| \leq C \kappa\left(\left(Q_{J}^{M} \cap E\right)_{N-M}\right) \leq C A_{0} 2^{-M d} \kappa\left(E_{N}\right)=C A_{0} \sigma\left(B_{J}^{M}\right) .
$$

To see (21), notice that

$$
\left\|R\left(\chi_{B_{J}^{M}} d \sigma\right)\right\|_{L^{\infty}\left(\mathbb{R}^{d}\right)} \leq C \frac{\kappa\left(E_{M}\right)}{\Lambda_{d-1}\left(\partial E_{M}\right)}\left\|R\left(\chi_{B_{J}^{M}} d \Lambda_{d-1}\right)\right\|_{L^{\infty}\left(\mathbb{R}^{d}\right)} \leq C .
$$

Since $\left\|R\left(\chi_{Q_{K}^{n}} d \nu\right)\right\|_{L^{\infty}\left(\mathbb{R}^{d}\right)} \leq C$, again by localization $([\mathrm{P}])$, in order to show (21) we only need to estimate the following differences for $0 \leq n<M$,

$$
R\left(b \chi_{Q_{K}^{n}} d \sigma\right)(x)-R\left(\chi_{Q_{K}^{n}} d \nu\right)(x)=\sum_{Q_{J}^{M} \subset Q_{K}^{n}} R \alpha_{J}^{M}(x),
$$

where $\alpha_{J}^{M}=\frac{\nu\left(Q_{J}^{M}\right)}{\sigma\left(B_{J}^{M}\right)} \chi_{B_{J}^{M}} d \sigma-\chi_{Q_{J}^{M}} d \nu$. Since $\int d \alpha_{J}^{M}=0,\left\|R \alpha_{J}^{M}\right\|_{L^{\infty}\left(\mathbb{R}^{d}\right)} \leq C$ and for $\left|x-c\left(B_{J}^{M}\right)\right|>c \sigma_{M}$,

(21) follows.

$$
\left|R\left(\alpha_{J}^{M}\right)(x)\right| \leq C \frac{\sigma_{M}^{d}}{\operatorname{dist}\left(x, Q_{J}^{M}\right)^{d}}
$$

At this point one can finish the proof by applying Theorem 7.1 of Volberg [Vo] with the function $b$, but we will give a direct argument based on [Ch1]. We thank the referee for the route through Theorem 7.1 of [Vo].

Given a cube $Q_{J}^{n}, 0 \leq n \leq M$, set

$$
\tilde{Q}_{J}^{n}=\bigcup_{B_{J}^{M} \cap Q_{J}^{n} \neq \emptyset} B_{J}^{M} .
$$

Notice that $\operatorname{diam}\left(\tilde{Q}_{J}^{n}\right)=c \sigma_{n} \approx \operatorname{diam}\left(Q_{J}^{n}\right)$ and $\sigma_{\mid Q_{J}^{n}}=\sigma_{\mid \tilde{Q}_{J}^{n}}$. By (19) and (20) we have

$$
\begin{aligned}
\sigma\left(\tilde{E}_{M} \backslash \bigcup_{\gamma} \tilde{P}_{\gamma}\right) & \geq \frac{1}{C} \int_{\tilde{E}_{M} \backslash \bigcup_{\gamma} \tilde{P}_{\gamma}}|b| d \sigma \\
& \geq \frac{1}{C}\left|\int_{\tilde{E}_{M}} b d \sigma\right|-\frac{1}{C} \sum_{\gamma}\left|\int_{P_{\gamma}} b d \sigma\right| \\
& >\frac{1}{C}\left(\epsilon_{0} \sigma\left(\tilde{E}_{M}\right)-\epsilon \sum_{\gamma} \sigma\left(P_{\gamma}\right)\right) .
\end{aligned}
$$

Therefore, for $\eta=\frac{\epsilon_{0}-\epsilon}{C-\epsilon}$,

$$
\sum_{\gamma} \sigma\left(P_{\gamma}\right) \leq(1-\eta) \sigma\left(\tilde{E}_{M}\right)
$$


We can now define our good set $G \subset \tilde{E}_{M}$. Set

$$
G=\tilde{E}_{M} \backslash \bigcup_{\gamma} \tilde{P}_{\gamma}
$$

By $(24), \eta \sigma\left(\tilde{E}_{M}\right) \leq \sigma(G) \leq \sigma\left(\tilde{E}_{M}\right)$. We want to construct the set $E^{\prime}$, by excising from $\tilde{E}_{M}$ the union of the stopping time cubes $\tilde{P}_{\gamma}$, and replacing each $\tilde{P}_{\gamma}$ by a union of two spheres. For each stopping time cube $\tilde{P}_{\gamma}$, set

$$
S_{\gamma}=\partial B_{\gamma}^{1} \cup \partial B_{\gamma}^{2}
$$

where $B_{\gamma}^{j}, j=1,2$ are two balls with center $c\left(S_{\gamma}\right):=c\left(B_{\gamma}^{1}\right)=c\left(B_{\gamma}^{2}\right) \in P_{\gamma}$ and such that

$$
2 \operatorname{diam}\left(B_{\gamma}^{1}\right)=\operatorname{diam}\left(B_{\gamma}^{2}\right)= \begin{cases}\frac{c}{2} \sigma_{n} & \text { if } P_{\gamma}=Q_{J}^{n}, \text { for some } 0 \leq n<M, \\ d_{M} & \text { if } P_{\gamma}=Q_{J}^{M} .\end{cases}
$$

Set

$$
E^{\prime}=G \cup \bigcup_{\gamma} S_{\gamma}=\left(\tilde{E}_{M} \backslash \bigcup_{\gamma} \tilde{P}_{\gamma}\right) \cup \bigcup_{\gamma} S_{\gamma},
$$

and define a measure $\sigma^{\prime}$ on $E^{\prime}$ as follows:

$$
\sigma^{\prime}=\left\{\begin{array}{l}
\sigma \quad \text { on } G \\
\frac{\sigma\left(P_{\gamma}\right)}{2}\left(\frac{\Lambda_{d-1 \mid \partial B_{\gamma}^{1}}}{\Lambda_{d-1}\left(\partial B_{\gamma}^{1}\right)}+\frac{\Lambda_{d-1 \mid \partial B_{\gamma}^{2}}}{\Lambda_{d-1}\left(\partial B_{\gamma}^{2}\right)}\right) \text { on } S_{\gamma} .
\end{array}\right.
$$

Using that $\sigma$ is doubling and has $(d-1)$-growth it is easy to see that $\sigma^{\prime}$ also satisfies these two properties.

For a system of dyadic cubes in $E^{\prime}$ satisfying the required properties (see Theorem 11 in [Ch1]), we take all cubes $\tilde{Q}_{J}^{n}, 0 \leq n \leq M$, which are not contained in any stopping time cube $\tilde{P}_{\gamma}$, together with each $S_{\gamma}$, together with each $\partial B_{\gamma}^{j}, j=1,2$ comprising $S_{\gamma}$, together with subsets of the two spheres,... and repeat.

We will now modify the function $b$ on the union $\cup_{\gamma} S_{\gamma}$ in order to obtain a new function $b^{\prime}$ defined on $E^{\prime}$, bounded and dyadic para-accretive with respect to the system of dyadic cubes defined above. Let

$$
b^{\prime}(x)=\left\{\begin{array}{l}
b(x) \quad \text { if } x \in G \\
g_{\gamma}(x)=c_{\gamma}^{1} \chi_{\partial B_{\gamma}^{1}}(x)-c_{\gamma}^{2} \chi_{\partial B_{\gamma}^{2}}(x) \quad \text { on } S_{\gamma},
\end{array}\right.
$$

where

$$
c_{\gamma}^{1}=2 \omega_{\gamma}, \quad c_{\gamma}^{2}=2 \omega_{\gamma}\left(1-\frac{\left|\nu\left(P_{\gamma}\right)\right|}{\sigma\left(P_{\gamma}\right)}\right) \text { and } \omega_{\gamma}=\left\{\begin{array}{l}
\frac{\nu\left(P_{\gamma}\right)}{\left|\nu\left(P_{\gamma}\right)\right|} \quad \text { if }\left|\nu\left(P_{\gamma}\right)\right| \neq 0 \\
1 \text { otherwise. }
\end{array}\right.
$$

Notice that the coefficients $c_{\gamma}^{j}, j=1,2$, are defined so that

$$
\int_{S_{\gamma}} g_{\gamma} d \sigma^{\prime}=\int_{P_{\gamma}} b d \sigma=\nu\left(P_{\gamma}\right)
$$


and $\left|c_{\gamma}^{1}\right|=2$ and $2(1-\epsilon) \leq\left|c_{\gamma}^{2}\right| \leq 2$, because $P_{\gamma}$ is a stopping time cube. The function $b^{\prime}$ is bounded because of the upper bound on the coefficients $c_{\gamma}^{j}, j=1,2$ and the fact that $\|b\|_{\infty} \leq C$.

For future reference, notice that, for every dyadic cube $Q$ in $E^{\prime}$, such that $Q \nsubseteq S_{\gamma}$ for all $\gamma$, there is a non-stopping time cube $Q^{*}\left(Q^{*}=\tilde{Q}_{K}^{n}\right.$ for some $\left.1 \leq n \leq M\right)$ uniquely associated to $Q$ by the identity

$$
Q=\left(Q^{*} \backslash \underset{\tilde{P}_{\gamma} \subset Q^{*}}{\bigcup} \tilde{P}_{\gamma}\right) \cup\left(\underset{\tilde{P}_{\gamma} \subset Q^{*}}{\bigcup} S_{\gamma}\right)
$$

Moreover one has $\operatorname{diam}(Q) \approx \operatorname{diam}\left(Q^{*}\right)$ and

$$
\sigma^{\prime}(Q)=\sigma\left(Q^{*}\right)-\sum_{\tilde{P}_{\gamma} \subset Q^{*}} \sigma\left(\tilde{P}_{\gamma}\right)+\sum_{\tilde{P}_{\gamma} \subset Q^{*}} \sigma^{\prime}\left(S_{\gamma}\right)=\sigma\left(Q^{*}\right)
$$

We will check now that, by construction, the function $b^{\prime}$ is dyadic para-accretive with respect to the system of dyadic cubes in $E^{\prime}$ :

If for some $\gamma, Q \subseteq S_{\gamma}$, the para-accretivity of $b^{\prime}$ follows from the definition of $g_{\gamma}$ and the lower bound on $\left|c_{\gamma}^{j}\right|, j=1,2$. Recall that, when examining the para-accretivity condition on $S_{\gamma}$, although identity (25) holds, we have a satisfactory lower bound on the integral over each child $\partial B_{\gamma}^{j}$ of $S_{\gamma}$, which turns to be enough for $b^{\prime}$ to be dyadic para-accretive.

Otherwise, let $Q^{*}$ be non-stopping time cube defined in (26). Then due to (25) and (27) we can write

$$
\left|\int_{Q} b^{\prime} d \sigma^{\prime}\right|=\left|\int_{Q^{*}} b d \sigma\right| \geq \epsilon \sigma\left(Q^{*}\right)=\epsilon \sigma^{\prime}(Q) .
$$

We must still show that $R\left(b^{\prime} \sigma^{\prime}\right)$ belongs to dyadic $B M O\left(\sigma^{\prime}\right)$. It is enough to show the following $L^{1}-$ inequality

$$
\left\|R\left(b^{\prime} \chi_{Q}\right)\right\|_{L^{1}\left(\sigma_{Q}^{\prime}\right)} \leq C \sigma^{\prime}(Q)
$$

for every dyadic cube in $E^{\prime}$.

Let $Q$ be some dyadic cube in $E^{\prime}$. We distinguish between two cases:

Case 1: For some $\gamma, Q \subseteq S_{\gamma}$. Then (28) follows from the boundedness of the coefficients $\left|c_{\gamma}^{j}\right|, j=1,2, \sigma\left(P_{\gamma}\right) \leq C \operatorname{diam}\left(P_{\gamma}\right)^{d-1}$ and $\Lambda_{d-1}\left(S_{\gamma}\right) \approx \operatorname{diam}\left(P_{\gamma}\right)^{d-1}$. 
Case 2: Otherwise, $Q=\left(Q^{*} \backslash \bigcup_{\tilde{P}_{\gamma} \subset Q^{*}} \tilde{P}_{\gamma}\right) \cup\left(\bigcup_{\tilde{P}_{\gamma} \subset Q^{*}} S_{\gamma}\right)$ for some non-stopping $Q^{*}=$ $\tilde{Q}_{K}^{n}, 1 \leq n \leq M$. Due to (25) we can write

$$
\begin{aligned}
R\left(b^{\prime} \chi_{Q}\right)(y) & =R\left(b \chi_{Q^{*}}\right)(y) \\
& +\sum_{\gamma: \tilde{P_{\gamma}} \subset Q^{*}} \int_{S_{\gamma}} g_{\gamma}(x)\left(K(x-y)-K\left(c\left(S_{\gamma}\right)-y\right)\right) d \sigma^{\prime}(x) \\
& +\sum_{\gamma: \tilde{P_{\gamma}} \subset Q^{*}} \int_{P_{\gamma}} b(x)\left(K\left(c\left(S_{\gamma}\right)-y\right)-K(x-y)\right) d \sigma(x) \\
& =A+B+C .
\end{aligned}
$$

By (21) (or (23) if $\left.Q^{*}=B_{J}^{M}\right), \| R\left(b \chi_{\left.Q^{*}\right)} \|_{L^{\infty}\left(\mathbb{R}^{d}\right)} \leq C\right.$. Hence

$$
\int_{Q}|A| d \sigma^{\prime} \leq C \sigma^{\prime}(Q)
$$

We deal now with term $B$. Set

$$
B 1=\int_{Q \backslash S_{\gamma}}\left|\int_{S_{\gamma}} g_{\gamma}(x)\left(K(x-y)-K\left(c\left(S_{\gamma}\right)-y\right)\right) d \sigma^{\prime}(x)\right| d \sigma^{\prime}(y)
$$

and

$$
B 2=\int_{S_{\gamma}}\left|\int_{S_{\gamma}} g_{\gamma}(x)\left(K(x-y)-K\left(c\left(S_{\gamma}\right)-y\right)\right) d \sigma^{\prime}(x)\right| d \sigma^{\prime}(y) .
$$

For $B 1$, let $g(Q) \in \mathbb{N}$ be such that $\operatorname{diam}(Q) \approx \sigma_{g(Q)}$ and $P_{\gamma}=Q_{J}^{n}$ for some $0 \leq n \leq M$. Observe that $\operatorname{diam}\left(S_{\gamma}\right) \approx \operatorname{diam}\left(P_{\gamma}\right) \approx \sigma_{n}$. Denote by $Q^{i}, g(Q) \leq i \leq n$, the cubes in $E^{\prime}$ contained in $Q$ and containing $S_{\gamma}$ such that $\operatorname{diam}\left(Q^{i}\right) \approx \sigma_{i}$ (note that the $Q^{i}$ are either $\tilde{Q}_{J}^{i}$ s or unions of spheres replacing the stopping time cubes of generation $i)$. Then by the boundedness of $g_{\gamma}$, the $(d-1)$-growth of $\sigma^{\prime}$ and the upper bound in (2),

$$
\begin{aligned}
B 1 & \leq C \sigma^{\prime}\left(S_{\gamma}\right) \sum_{i=g(Q)}^{n-1} \int_{Q^{i} \backslash Q^{i+1}} \frac{\sigma_{n}}{\sigma_{i}^{d}} d \sigma^{\prime} \\
& \leq C \sigma^{\prime}\left(S_{\gamma}\right) \sum_{i=g(Q)}^{n-1} \frac{\sigma_{n}}{\sigma_{i}} \leq C \sigma^{\prime}\left(S_{\gamma}\right) \sum_{i} 2^{-i} \leq C \sigma^{\prime}\left(S_{\gamma}\right) .
\end{aligned}
$$

For $B 2$ argue like in the previous case, i.e. (28) for $Q=S_{\gamma}$, to get that $B 2 \leq C \sigma^{\prime}\left(S_{\gamma}\right)$. Therefore by $\sigma^{\prime}\left(S_{\gamma}\right)=\sigma\left(P_{\gamma}\right)$, the packing condition (24) (with $\tilde{E}_{M}$ replaced by $Q^{*}$ ) and (27) we get that $\int_{Q}|B| d \sigma^{\prime} \leq C \sigma^{\prime}(Q)$.

Similar arguments work to show $\int_{Q}|C| d \sigma^{\prime} \leq C \sigma^{\prime}(Q)$. Therefore we are done. 


\section{Acknowledgements}

J. G. and L. P. were supported in part by NSF Grant DMS-0402720. L. P. was supported by a Fulbright/MECD scholarship from Secretaría de Estado de Educación y Universidades and Fondo Social Europeo. L. P. and X. T. were also partially supported by grants MTM2004-00519, Acción Integrada HF2004-0208 (Ministerio de Ciencia y Tecnología) and SGR-2005-00774 (Generalitat de Catalunya).

\section{References}

[Ch1] M. Christ, A T(b) Theorem with remarks on analytic capacity and the Cauchy integral, Colloq. Math. 60/61 (1990), 601-628.

[Ch2] _ Lectures on singular integral operators, CBMS Regional Conference Series in Mathematics 77, Providence, RI, 1990.

[DØ] A. M. Davie and B. Øksendal, Analytic capacity and differentiability properties of finely harmonic functions, Acta Math. 149 (1982) 127-152.

[GV] J. Garnett and J. Verdera, Analytic capacity, bilipschitz maps and Cantor sets, Math. Res. Lett. 10 (2003), no. 4, 515-522.

$[\mathrm{MPrV}]$ J. Mateu, L. Prat, and J. Verdera, The capacity associated to signed Riesz kernels, and Wolff potentials, J. Reine Angew. Math. 578 (2005) 201-223.

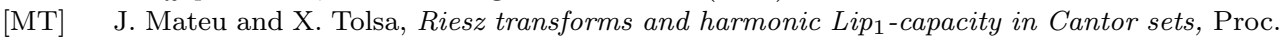
London Math. Soc. 89 (2004), no. 3, 676-696.

[MTV] J. Mateu, X. Tolsa and J. Verdera, The planar Cantor sets of zero analytic capacity and the local $T(b)$ theorem, J. Amer. Math. Soc. 16 (2003), no. 1, 19-28.

[P] P. V. Paramonov, Harmonic approximations in the $\mathcal{C}^{1}$-norm, Math. USSR-Sb. 71 (1992) 183-207.

[T1] X. Tolsa, Painlevé's problem and the semiadditivity of analytic capacity, Acta Math. 190 (2003) 105-149.

[T2] Bilipschitz maps, analytic capacity and the Cauchy integral, Ann. of Math. 162 (2005) 1241-1302.

[Vo] A. Volberg, Calderón-Zygmund capacities and operators on nonhomogeneous spaces, CBMS Regional Conference Series in Mathematics, 100, Providence, RI, 2003.

Department of Mathematics, UClA, Los Angeles, CA 90095

E-mail address: jbg@math.ucla.edu

Departament de Matemàtica Aplicada i Anàlisi, Universitat de Barcelona, Catalunya

E-mail address: lprat@ub.edu

iCREA and Departament de Matemàtiques, Universitat Autònoma de Barcelona, CATALUNYa

E-mail address: xavier.tolsa@uab.cat 